S. DESHMUKH

KODAI MATH. J.

11 (1988), 155-158

\title{
REDUCTION IN CODIMENSION OF MIXED FOLIATE $C R$-SUBMANIFOLDS OF A KAEHLER MANIFOLD
}

\author{
By SHARIEF DEShMUKH
}

\begin{abstract}
Starting with a proper mixed foliate $C R$-submanifold $M$ of dimension $2 p+q$ in a hyperbolic complex space form $\bar{M}(-4)$ of dimension $m(m \geqq 2 p+2 q)$, it has been proved that under a suitable condition on second fundamental form there exists a $(2 p+2 q)$-dimensional totally geodesic submanifold $M^{\prime}$ of $\bar{M}$ such that $M$ is a mixed foliate $C R$-submanifold of $M^{\prime}$.
\end{abstract}

1. Let $\bar{M}$ be an $m$-dimensional hyperbolic complex space form, that is, a Kaehler manifold of constant holomorphic sectional curvature -4 . The curvature tensor $\bar{R}$ or $\bar{M}$ is given by

$$
\begin{array}{r}
\bar{R}(X, Y) Z=-\{g(Y, Z) X-g(X, Z) Y+g(J Y, Z) J X \\
-g(J X, Z) J Y+2 g(X, J Y) J Z\},
\end{array}
$$

where $J$ is the almost complex structure of $\bar{M}$ and $g$ is the hermitian metric.

$A(2 p+q)$-dimensional submanifold $M$ of $\bar{M}$ is called a $C R$-submanifold if there exists a pair of orthogonal complementary distributions $D$ and $D^{\perp}$ such that $J D=D$ and $J D^{\perp} \subset \nu$, where $\nu$ is the normal bundle of $M$ and $\operatorname{dim} D=2 p$, $\operatorname{dim} D^{\perp}=q$, [1]. A $C R$-submanifold is said to be proper if neither $D=\{0\}$ nor $D^{\perp}=\{0\}$. We shall denote by $\bar{\nabla}, \nabla, \nabla^{\perp}$ the Riemannian connections on $\bar{M}, M$, and the normal bundle respectively. They are related by the Gauss and Weingarten formulae

$$
\bar{\nabla}_{X} Y=\nabla_{X} Y+h(X, Y), \quad \bar{\nabla}_{X} N=-A_{N} X+\nabla_{\bar{X}}^{\frac{1}{X}} N, \quad N \in \nu
$$

where $h(X, Y)$ and $A_{N} X$ are second fundamental forms and $g(h(X, Y), N)$ $=g\left(A_{N} X, Y\right)$.

A $C R$-submanifold $M$ is said to be mixed foliate if (i) $D$ is integrable, and (ii) $h(X, Y)=0, X \in D, Y \in D^{\perp}$. Mixed foliate $C R$-submanifolds have been studied by A. Bejancu [1] and B. Y. Chen [2]. It is known that if $M$ is a mixed foliate $C R$-submanifold of a complex space form $\bar{M}(c)$, then $c<0$, that is why we consider mixed foliate $C R$-submanifolds of $\bar{M}(-4)$. In a mixed foliate $C R$-submanifold of a Kaehler manifold the following hold good [2]

Received May 2, 1987 


$$
\begin{aligned}
& h(X, J Y)=h(J X, Y), \quad X, Y \in D \\
& A_{N} X \in D, \quad X \in D \quad \text { and } A_{N} X \in D^{\perp}, \quad X \in D^{\perp} \\
& A_{N} J X=-J A_{N} X, \quad X \in D \\
& A_{J X} Y=A_{J Y} X, \quad X, Y \in D^{\perp} .
\end{aligned}
$$

The normal bundle splits as $\nu=J D^{\perp} \oplus \mu$, where $\mu$ is a $J$-invariant sub-bundle of $\nu$.

For a submanifold $M$, the first normal space $N_{p}^{1}$ and the first osculating space $O_{p}^{1}$ at $p \in M$ are defined by

$$
N_{p}^{1}=\left\{h_{p}\left(X_{p}, Y_{p}\right): X_{p}, Y_{p} \in T_{p} M\right\} \quad \text { and } \quad O_{p}^{1}=T_{p} M \oplus N_{p}^{1},
$$

where $T_{p} M$ is the tangent space of $M$ at $p$. A subspace $V$ of $T_{p} \bar{M}$ is said to define a Lie-triple system if $\bar{R}_{p}\left(X_{p}, Y_{p}\right) Z_{p} \in V$ for $X_{p}, Y_{p}, Z_{p} \in V$. For a Lietriple system $V$ in a symmetric space $\bar{M}$, there exists a unique complete totally geodesic submanifold $M^{\prime}$ of $\bar{M}$ such that $T_{p} M^{\prime}=V$, (cf. [6]).

The equations of Gauss, Codazzi and Ricci are

$$
\begin{aligned}
& R(X, Y ; Z, W)= \vec{R}(X, Y ; Z, W)+g(h(X, Z), h(Y, W)) \\
&-g(h(Y, Z), h(X, W)) \\
& {[\bar{R}(X, Y) Z]^{\perp}=\left(\bar{\nabla}_{X} h\right)(Y, Z)-\left(\bar{\nabla}_{Y} h\right)(X, Z) } \\
& \bar{R}\left(X, Y ; N_{1}, N_{2}\right)=R^{\perp}\left(X, Y ; N_{1}, N_{2}\right)-g\left(\left[A_{N_{1}}, A_{N_{2}}\right](X), Y\right),
\end{aligned}
$$

where []$^{\perp}$ denotes the normal component, $R^{\perp}$ is the curvature tensor of $\nabla^{\perp}$, and $\left(\bar{\nabla}_{X} h\right)(Y, Z)=\nabla_{X}^{\frac{1}{X}} h(Y, Z)-h\left(\nabla_{X} Y, Z\right)-h\left(Y, \nabla_{X} Z\right)$.

2. Let $M$ be a mixed foliate $C R$-submanifold of $\bar{M}(-4)$. Then it follows that (cf. [2]).

$$
\begin{aligned}
& \nabla_{X} Y D^{\perp}, \quad X, Y \in D^{1} ; \nabla_{X} Y \in D, \quad X \in D^{\perp}, \quad Y \in D \text { and } \\
& \nabla_{X}^{1} J Y \in J D^{1}, \quad X \in D, \quad Y \in D^{\perp} .
\end{aligned}
$$

PROPOSITION 2.1. Let $M$ be a proper mixed foliate $C R$-submanifold of hyperbolic complex space form $\bar{M}(-4)$. Then $h(X, Y) \in J D^{1}, X, Y \in D$.

Proof. For $X, Y \in D$ and $Z \in D^{\perp}$, the equation (1.1) gives

$$
[\bar{R}(X, Y) Z]^{\perp}=-2 g(X, J Y) J Z \text {. }
$$

Using this in equation (1.8) and noting that $h([X, Y], Z)=0$, we get

$$
-2 g(X, J Y) J Z=h\left(X, \nabla_{Y} Z\right)-h\left(Y, \nabla_{X} Z\right) .
$$

Taking the inner product with $J W \in J D^{\perp}$ and replacing $X$ by $J X$ we get 


$$
2 g(X, Y) g(Z, W)=g\left(A_{J W} X, A_{J Z} Y\right)+g\left(A_{J Z} X, A_{J W} Y\right),
$$

where we have used $A_{J W} X, J A_{J W} X \in D$ and the equations (1.2), (1.5).

Also for $X, Y \in D, Z \in D^{\perp}$ and $N \in \mu$, using (1.1) and (1.9), we get

$$
g\left(\left[A_{J Z}, A_{N}\right](X), Y\right)=0,
$$

where we use the fact $R^{\perp}(X, Y) J Z \in J D^{\perp}$ by virtue of (2.1). Taking inner product with $N \in \mu$ in (2.3), we get with similar techniques as in (2.4) that

$$
g\left(A_{N} X, A_{J Z} Y\right)+g\left(A_{J Z} X, A_{N} Y\right)=0 .
$$

Combination of (2.5) and (2.6) gives $A_{J Z}\left(A_{N} X\right)=0, X \in D$. Since, for every $X \in D, A_{N} X \in D$, replacing $X$ by $A_{N} X$ in (2.4) and using $A_{J Z}\left(A_{N} X\right)=A_{J W}\left(A_{N} X\right)$ $=0$, we get

$$
2 g\left(A_{N} X, Y\right) g(Z, W)=0
$$

and $M$ being a proper $C R$-submanifold we get $g(h(X, Y), N)=0$.

COROLlARY 2.1. Let $M$ be a proper mixed foliate $C R$-submanifold of a hyperbolic complex space form $\bar{M}(-4)$. Then

(a) $A_{J Z}^{2} X=X, X \in D$ and $Z$ a unit vector in $D^{1}$

(b) $A_{J Z} A_{J W} X=-A_{J Z} A_{J Z} X, X \in D$ and $Z \perp W, Z, W \in D^{\perp}$.

The proof follows from equation (2.4).

LEMMA 2.1. Let $M$ be a mixed foliate $C R$-submanifold of $\bar{M}(-4)$. Then for $X, Y, Z \in X(M)$, the Lie-algebra of vector fields on $M$

$$
\bar{R}_{p}\left(X_{p}, Y_{p}\right) Z_{p}, \bar{R}_{p}\left(J X_{p}, Y_{p}\right) Z_{p}, \bar{R}\left(J X_{p}, J Y_{p}\right) J Z_{p} \in T_{p} M \oplus J D_{p}^{1}
$$

for $p \in M$.

The proof is trivial and follows at once from (1.1).

LEMMA 2.2. Let $M$ be a proper mixed foliate $C R$-submanifold of $\bar{M}(-4)$ satisfying $h(X, Y) \in J D^{\perp}$ for $X, Y \in D^{\perp}$. Then $T_{p} M \oplus J D_{p}^{\perp}$ is the first osculating space at $p \in M$.

Proof. It suffices to prove

$$
J D^{1}=\{h(X, Y): X, Y \in *(M)\} .
$$

From proposition 2.1 it follows that $\{h(X, Y): X, Y \in X(M)\} \subset J D^{\perp}$. Now suppose there exists a unit vector $J Z \in J D^{\perp}$ such that $g(h(X, Y), J Z)=0$ for all $X, Y \in X(M)$. This gives $g\left(A_{J Z} X, Y\right)=0$ for all $Y$. In particular we have for $X \in D$ and $Y=A_{J Z} X, g\left(A_{J Z} X, A_{J Z} X\right)=0$. Which is impossible by Corollary 1.1 (a), unless $M$ is totally real (that is $D=\{0\}$ ). Hence we get the quality. 
Now we prove the main result.

THEOREM. Let $M$ be $a(2 p+q)$-dimensional proper mixed foliate $C R$-submanifold of the hyperbolic complex space form $\bar{M}(-4)$ of dimension $m(m \geqq 2 p+2 q)$ satisfying $h(X, Y) \in J D^{\perp}$ for $X, Y \in D^{\perp}$. Then there exists a complete totally geodesic complex submanifold $M^{\prime}$ of dimension $2 p+2 q$ of $\bar{M}$ such that $M$ is a mixed foliate CR-submanifold of $M^{\prime}$.

Proof. By Lemmas 2.1 and 2.2, it follows that the osculating space $O_{p}^{1}$ is a Lie-triple system at each point $p \in M$. Hence there exists a complete totally geodesic submanifold $M^{\prime}$ of $\bar{M}(-4)$ of dimension $=\operatorname{dim} O_{p}^{1}=2 p+2 q$. By a result of Chen and Ogiue [4], submanifolds of complex space forms with parallel second fundamental form are either complex or totally real. Since $M^{\prime}$ is totally geodesic it satisfies the hypothesis of this Theorem, and therefore $M^{\prime}$ is either a complex or totally real submanifold. But owing to the presence of the nontrivial $J$-invariant $D$, it cannot be totally real. In fact $M^{\prime}$ is a hyperbolic complex space form, and $M$ is a mixed foliate $C R$-submanifold of $M^{\prime}(-4)$.

\section{REFERENCES}

[1] A. Bejancu, $C R$-submanifold of a Kaehler manifold, I, II, Proc. Amer. Math. Soc. 69 (1978), 135-142, Trans. Amer. Math. Soc. 250 (1979), 333-345.

[2] B. Y. CHEN, CR-submanifolds of a Kaehler manifold II, J. Diff. Geom. 16 (1981), 493-509.

[3] B. Y. Chen, Geometry of Submanifolds and its Applications, Science Univ. Tokyo, (1981).

[4] B.Y. Chen And K. OGIue, On totally real submanifolds, Trans. Amer. Math. Soc. 193 (1975), 257-266.

[5] J. Erbacher, Reduction of codimension of an isometric immersion, J. Diff. Geom. 5 (1971), 333-340.

[6] S. Helgason, Differential Geometry and Symmetric spaces, Academic Press, New York (1962).

Department of Mathematics

King Saud University

P. O. Box 2455, RiYadH 11451

Saudi Arabia 